\title{
HISTORIAS DE VIDA Y MOVILIDADES ACADÉMICAS DE INMIGRANTES
}

\author{
- JOSÉ GONZÁLEZ-MONTEAGUDO \\ https://orcid.org/0000-0002-3094-8092 \\ Universidad de Sevilla \\ WILTON CARLOS LIMA DA SILVA \\ https://orcid.org/0000-0002-1507-8017 \\ Universidade Estadual Paulista “Julio de Mesquita Filho"
}

RESUMEN Este artículo se centra en la migración y la movilidad académica. Se basa en un estudio de caso biográfico sobre un investigador universitario de origen inmigrante. La primera sección desarrolla el contexto de las cuestiones abordadas, destacando las lagunas actuales en la investigación sobre la movilidad académica de los migrantes. La sección metodológica proporciona una rápida justificación del enfoque biográfico utilizado para producir y construir la narrativa del caso, que se presenta en la siguiente sección. Se comentan varias dimensiones importantes sobre el caso presentado, referidas a la cooperación, los contextos transfronterizos y multilingües, el diálogo, la perspectiva histórica y comparativa, y las universidades inclusivas. Con ello, se intenta contribuir a la construcción de una perspectiva eco-formación, basada en la movilidad intercultural. Se destacan los beneficios de las metodologías cualitativas y narrativas para conocer y comprender mejor las cuestiones referidas a las migraciones, las identidades y las diversidades interculturales.

Palabras clave: Historias de vida. Migraciones. Movilidades académicas. Interculturalidad. Aprendizaje informal.

\section{ABSTRACT LIFE HISTORIES AND ACADEMIC MOBILITIES OF IMMIGRANTS}

This article focuses on migration and academic mobility. It is based on a biographical case study of an university researcher from immigrant origin. The first section develops the literature review of the issues addressed, highlighting current gaps in research on the academic mobility of migrants. The methodological section provides a justification of the biographical approach used to produce and con- 
struct the narrative case, which is presented in the following section. Several important dimensions of the presented case are discussed, referring to cooperation, cross-border and multilingual contexts, dialogue, historical and comparative perspective, and inclusive universities. The aim is to contribute to the construction of an eco-training perspective, based on intercultural mobility. The benefits of qualitative and narrative methodologies are highlighted, to better know and understand the issues of migrations, identities and intercultural diversities.

Keywords: Life histories. Migration. Academic mobilities. Interculturality. Informal learning.

\section{RESUMO}

\section{HISTÓRIAS DE VIDA E MOBILIDADES ACADÉMICAS DOS IMIGRANTES}

Este artigo centra-se na migração e na mobilidade académica. Baseia-se num estudo de caso biográfico de um investigador universitário de origem imigrante. A primeira secção desenvolve a revisão bibliográfica das questões abordadas, destacando as lacunas atuais da investigação sobre a mobilidade académica dos migrantes. A secção metodológica fornece uma justificação da abordagem biográfica utilizada para produzir e construir o caso narrativo, que é apresentada na secção seguinte. São discutidas várias dimensões importantes do caso apresentado, referindo-se à cooperação, aos contextos transfronteiriços e multilíngues, ao diálogo, à perspectiva histórica e comparativa e às universidades inclusivas. O objetivo é contribuir para a construção de uma perspectiva de eco-formação, baseada na mobilidade intercultural. Destacam-se os beneficios das metodologias qualitativas e narrativas para melhor conhecer e compreender as questões das migrações, identidades e diversidades interculturais.

Palavras-chave: Histórias de vida. Migração. Mobilidade académica. Interculturalidade. Aprendizagem informal.

\section{Migraciones y movilidades académicas}

En los últimos años, la migración y la movilidad se han generalizado. La globalización económica, los nuevos medios de transporte, las crecientes desigualdades, los conflictos y las guerras han dado lugar a flujos migratorios internacionales que han afectado a todos los países del mundo. Los migrantes, los viajeros y los estudiantes internacionales están dando lugar a nuevas formas de movilidad e interdependencia, al desarrollo de las empresas transnacionales, al aumento de la diversidad cultural y a formas recientes de cooperación bilateral, regional y mundial (UCKER, 2018). En 2007, las Naciones Unidas estimaron que ha- 
bía 200 millones de migrantes internacionales, definidos como personas que habían vivido al menos un año fuera de su país de nacimiento. Esto representaba alrededor del $3 \%$ de los 6.500 millones de habitantes del mundo (CASTLES y MILLER, 2009, p. 2-7). Esta migración debe considerarse como parte de un fenómeno global, complejo y cambiante que afecta tanto a los que migran como a los que reciben a los migrantes, y que transforma las condiciones sociales y culturales de todos los ciudadanos.

Este artículo explora el tema de los migrantes académicos de origen mexicano (o más ampliamente latinoamericano) que trabajan en universidades de los Estados Unidos. Para enfocar mejor nuestro tema, presento aquí algunos datos y puntos de referencia sobre los movimientos migratorios que tienen lugar en los Estados Unidos (como país anfitrión). Entre 1850 y 1914, la mayoría de los migrantes que llegaron a Estados Unidos procedían de Irlanda, Italia, España y Europa del Este, zonas donde la industrialización comenzó más tarde. Los Estados Unidos de América propusieron el sueño de convertirse en un agricultor o comerciante independiente en la Nueva Tierra de las Oportunidades; el período más intenso fue entre 1861 y 1920, cuando llegaron 30 millones de personas (CASTLES y MILLER, 2009, p. 84).

En las últimas décadas, la inmigración total, referida a extranjeros con residencia legal permanente en Estados Unidos, fue de 4,5 millones en el período 1971-1980, cifra que ascendió a 7,3 millones entre 1981-1990, y aumentó a 9,1 millones entre 1990-2000. Por país, el mayor número de migrantes que obtuvieron la ciudadanía en Estados Unidos provenía de México, India y Filipinas (CASTLES y MILLER, 2009, p. 120). Para México, la población de México a Estados Unidos aumentó de 2.2 millones en 1980 a 11.9 millones en 2010. Los Estados Unidos tienen los niveles más altos de inmigración en el mundo. Entre estos migrantes, los mexicanos son el grupo mayoritario, con el $27.6 \%$ de la población total del país norteamericano. (DELGADO WISE, 2013; DELGADO WISE y CHÁVEZ, 2016).

La internacionalización de las universidades y la movilidad académica se presentan a menudo como políticas ideológicamente neutrales, coherentes e inconexas. La conceptualización de las personas como recursos que deben trasladarse y reubicarse en todo el mundo con una atención limitada a la identidad, la comunidad, el cuidado o el dominio emocional tiene implicaciones sociales, éticas, financieras y de equidad. Urry (2007, p.12) argumenta que la movilidad ha sido una "caja negra para las ciencias sociales", pues ha sido considerada generalmente un conjunto de procesos neutrales. A menudo se minimiza el significado y las consecuencias de las experiencias profundas, incluso corporales, derivadas de la movilidad. Por otro lado, en el ámbito de la educación superior, observamos una falta de estudios de sociología y etnografia sobre la movilidad. La literatura sobre movilidad estudiantil en la academia global está muy desarrollada (GONZÁLEZ-MONTEAGUDO, 2016; CHANG, 2011 y 2015; UCKER, 2018). Sin embargo, sólo unos pocos estudios exploran las perspectivas y testimonios de la movilidad de los profesores e investigadores migrantes (KIM, 2010). El paradigma de la nueva movilidad está empezando a hacerse notar, dentro de una sociología dinámica y abierta (SHELLER y URRY, 2006; SHELLER, 2014).

La movilidad académica que se estudia en este artículo implica trasladarse del Sur Global al Norte Global por razones económicas, políticas o de derechos humanos. Este es generalmente el caso de los académicos latinoamericanos que viven y trabajan en los Estados Unidos. Para ellos, la migración es vista como un compromiso a largo plazo (DELGADO WISE, CHÁVEZ y RODRÍGUEZ, 2016). 
Utilizando la historia producida en el contexto de entrevistas biográficas con un profesor-investigador nacido en México, que actualmente trabaja en una universidad de Texas (Estados Unidos), este artículo intenta contribuir a una mejor comprensión, desde la perspectiva de los actores involucrados, de los retos y oportunidades asociados con la migración como un viaje transnacional, intercultural y transformador.

\section{Metodología}

Este estudio de caso se realizó en el contexto de una investigación sobre los migrantes latinoamericanos que trabajan en universidades de los Estados Unidos.

Las entrevistas con este profesor-investigador mexicano, realizadas por uno de los coautores, se realizaron de acuerdo a un enfoque cualitativo y narrativo, pero incluyeron también preguntas semiestructuradas, para enfocar mejor los matices específicos. Para comprender mejor las experiencias y perspectivas sobre la movilidad y la internacionalización, se exploraron los siguientes temas: el contexto familiar y social, el itinerario educativo, la historia de la migración y la movilidad, las motivaciones para la movilidad, las ventajas y desventajas personales y profesionales de la movilidad internacional, los factores que han contribuido a que las experiencias de movilidad sean positivas o negativas, las experiencias sociales y el perfil como profesor e investigador académico.

Las entrevistas se llevaron a cabo durante una estancia de investigación realizada por uno de los coautores en Estados Unidos de América en 2015. Aunque las entrevistas se realizaron en inglés, Ezequiel utilizó el español muchas veces, particularmente en referencia a la familia, sus raíces mexicanas y temas de identidad, cultura y desarrollo. Este cambio constante entre el inglés y el español es uno de los rasgos distintivos de esta entrevista y refleja intensamente los problemas de la diversidad cultural, el cruce de fronteras, la hibridación y el bilingüismo.

Las entrevistas prestaron especial atención a la narración de experiencias, perspectivas personales, contextos culturales y profesionales, trayectorias profesionales y la interacción de la internacionalización con identidades particulares. Las entrevistas se transcribieron y fueron codificadas temáticamente. Los análisis de las entrevistas biográficas exploran la dialéctica entre las dimensiones personales y las estructuras sociales, tratando de explicar y comprender las dinámicas que vinculan la subjetividad con la sociedad y la cultura (GONZÁLEZ-MONTEAGUDO, 2016).

Para construir la narrativa del caso, se decidió contar la perspectiva del sujeto de una manera cercana al contenido de la transcripción de la entrevista. Esperamos que esta elección transmita algo de la frescura y autenticidad que se encuentran en los testimonios de este investigador.

El participante, al que nombraremos como Ezequiel para mantener el anonimato, se mostró muy interesado en colaborar en nuestra investigación. Ofreció una narración muy completa y rica de detalles. Se mostró muy feliz al poder compartir con uno de los coautores su itinerario en el sentido amplio del término, lo que para él incluyó numerosas referencias a sus raices familiares, al contexto social y cultural de México, a los espacios y tiempos de su infancia, a su carrera educativa y profesional, a su compromiso como militante social y a su perfil académico rebelde y alternativo. Para favorecer la expresión de una voz auténtica por parte de Ezequiel, uno de nosotros, como entrevistador, aceptó compartir durante las entrevistas ideas y experiencias cercanas a la sensibilidad del participante. Esto permi- 
tió construir un diálogo sincero y fluido, reconociendo la importancia, al mismo tiempo, de la voz del participante y del investigador. Para nosotros, esto significa intentar superar la tentación 'extractivista` que a veces está presente en los enfoques biográficos y narrativos. Estas es una cuestión de profundo calado, sobre la cual reflexionó Ezequiel durante las entrevistas. En más de una ocasión habló de los roles que podemos desempeñar, como investigadores, acompañadores e incluso colonizadores. Este tema no constituye el foco central de nuestro artículo, pero visibiliza en la narrativa de Ezequiel el debate ideológico sobre qué significa investigar desde el punto de vista epistemológico, ético, ideológico y político.

\section{El itinerario de Ezequiel: un caso} satisfactorio de inclusión social y profesional en ámbito académico

\section{de un inmigrante}

Ezequiel, de origen mexicano, es un líder comunitario y profesor-investigador en una universidad de Texas. El caso gira en torno a las ideas centrales del cruce de fronteras, la mezcla cultural, la construcción de la identidad cultural de los grupos minoritarios y las pedagogías críticas en la educación formal y el desarrollo comunitario. Para introducir el caso, comenzamos describiendo las raíces familiares de Ezequiel, siempre a partir de su testimonio durante las entrevistas.

El padre de Ezequiel nació en 1936 en el estado mexicano de Nuevo León y tenía 10 hermanos. El padre fue pastor durante su infancia. Su padre y los hermanos de su padre crecieron durante el período en que se creó el Programa Bracero. Este programa consistió en un acuerdo entre los gobiernos de los Estados Unidos y México para promover la contratación de trabajadores agrícolas mexicanos, para reemplazar a los trabajadores estadounidenses que participaron en la Segunda Guerra Mundial, en base a las necesidades laborales de las diferentes temporadas agrícolas. Contratados como trabajadores agrícolas, fueron llamados mojados, un término que se refiere a los migrantes irregulares e indocumentados que cruzan la frontera para trabajar temporalmente en los Estados Unidos.

La madre de Ezequiel era de cultura mexicana, pero ella nació y vivió al norte de la frontera. Como resultado, ella era ciudadana de los Estados Unidos. Debido a la actividad ilegal del abuelo materno como contrabandista, la familia tuvo que huir de los Estados Unidos y esconderse en México.

El padre y la madre de Ezequiel se conocieron en México, se casaron y tuvieron cuatro hijos. En 1968, toda la familia tuvo que abandonar México y establecerse en Texas, a cuarenta kilómetros al norte de la frontera. Ezequiel nació en México en 1963. Comenzó a vivir en los Estados Unidos cuando tenía cinco años. Inicialmente los padres sólo hablaban español y frecuentemente cruzaban la frontera en ambas direcciones, principalmente para visitar a parientes que aún vivían en México. Ezequiel resume sus antecedentes familiares expresando que él y sus tres hermanos eran niños transnacionales, con una cultura transfronteriza. El relato sobre el momento del cruce de la frontera y sus implicaciones es muy elocuente:

La vida de mi padre había sido amenazada, y él [...] yo, como padre que soy, puedo imaginarme esto, ¿verdad? Todo pasando por su mente y diciendo: "vale, entonces si me pasa algo, está mi mujer, dejo a mi mujer sola con tres niños pequeños, y entonces [...] ¿Qué tipo de vida van a vivir?". Así que él y mi madre están de acuerdo en cruzar la frontera hacia los Estados Unidos [...] Mis padres tenían inteligencia natural y también inteligencia comunitaria. Ellos sabían cómo transitar por los sistemas. Y, así, el último día de 1968, mis padres recogieron a la familia y cruzamos el río. $Y$ nos establecimos en Texas, a 
unos 25 kilómetros al norte de donde nacimos y crecimos. El lugar no estaba muy alejado, pero, culturalmente, ya sabes, lingüísticamente, y la moneda... era un mundo totalmente diferente.

Ezequiel destaca la importancia de preservar el español y no perderlo como condición para mantener la identidad mexicana. Ezequiel y sus hermanos fueron a una escuela pública en Texas, en un ambiente que él describe como muy "mexicano". Ezequiel insiste en la importancia que el padre da a la educación de los hijos. Ezequiel expresó con orgullo que los cuatro hermanos han obtenido cuatro títulos universitarios, tres maestrías y dos doctorados. Cabe destacar que Ezequiel ha seguido una trayectoria educativa y profesional muy cercana a la de su hermano Ruperto, nacido en 1964, y que los dos hermanos han trabajado juntos en numerosos proyectos educativos y de desarrollo.

Ezequiel subraya la importancia de los valores aportados a la familia y a la comunidad, elementos que influyeron decisivamente en él y en su hermano Ruperto: "El origen de nuestra educación es la familia y la comunidad". Ezequiel y Ruperto estudiaron en la década de 1980 en una universidad de Austin, la capital del estado. Ezequiel recuerda que la formación fue buena, pero Ezequiel señala que no aprendieron nada sobre sí mismos, porque la universidad le dio la espalda a la diversidad cultural y social:

En los años 80 mi hermano y yo éramos estudiantes universitarios en Austin. En la universidad éramos unos 48.000 estudiantes, pero sólo había como unos 2.000 como nosotros, que éramos mexicanos. $Y$ casi no había profesores mexicanos, ¿verdad? Así que no veíamos la literatura latinoamericana que nos atraía. Así que estudiamos de todo, excepto a nosotros mismos, ¿verdad?

A mediados de los años ochenta, esta fue una de las razones por las que los hermanos
Ezequiel y Ruperto, durante sus estudios universitarios, animaron al padre a escribir su autobiografia. Esta historia debe estar basada en historias, recuerdos y anécdotas que el padre solía contar. Este proceso de escritura autobiográfica del padre fue un incidente crítico que influyó profundamente en el futuro viaje personal y profesional de Ezequiel.

Durante este proceso, una nueva conciencia surgió en él y comenzó a desarrollar un proceso de aprendizaje de gran alcance. El hábito del padre de usar anécdotas e historias como herramientas educativas animó a Ezequiel y a su hermano Ruperto a explorar cómo el diálogo podría redefinirse en contextos educativos y comunitarios. En efecto, para Ezequiel, el diálogo, concebido como una dimensión transversal de la existencia humana, tiene implicaciones en tres contextos diferentes: investigación, educación y desarrollo comunitario. La experiencia de Ezequiel en la escuela primaria, secundaria, preparatoria y universidad le confirmó que el conocimiento sobre el mundo social de los estudiantes era muy escaso.

Para intervenir al mismo tiempo desde el punto de vista educativo y político, Ezequiel destaca la necesidad de trabajar en la formación de la identidad personal y colectiva, ayudando a la comunidad mexicana a ser reconocida como uno de los principales actores de su propio progreso. Esto lleva a Ezequiel a reconocer la necesidad de desarrollar el trabajo político, promoviendo el lenguaje y la crítica como medio de empoderamiento y agencia. Ezequiel enfatiza continuamente a lo largo de la entrevista que su forma de entender y practicar la investigación proviene de las historias y experiencias heredadas de sus padres y familiares.

Ezequiel resaltó que la migración y el cruce de fronteras son, al mismo tiempo, cuestiones culturales, políticas y técnicas. Esto también implica hablar de ideología y de geografia, 
pero en continuidad con lo aprendido en el ámbito familiar y comunitario:

Así que el cruce de la frontera para nosotros fue tanto cultural como político, y también técnico. $\mathrm{Y}$ todo el cruce de la frontera y la experiencia migratoria es tanto ideológico como geográfico. Y se convirtió en algo que empezamos a utilizar como una estrategia para el desarrollo, como una estrategia para el crecimiento, pero también como una estrategia para el empoderamiento, porque, cuando éramos estudiantes en la universidad en Austin, podíamos lograr tanto porque entendíamos dos idiomas. $Y$ eso dio origen a un cierto proceso de investigación que fue realmente distinto y muy importante, porque, para nosotros, la investigación se originó de nuestras historias y las experiencias que tuvimos de nuestros parientes. $Y$ luego pasó a la ciencia.

La falta de capital social y económico, un tema que Ezequiel destacó en varias ocasiones, ha sido compensado por el compromiso con su propia comunidad y el territorio. Esta hibridación, típica de los contextos interculturales y transfronterizos, ha fomentado un enfoque muy inclusivo de la enseñanza, el aprendizaje, la investigación y el desarrollo comunitario, basado en una atención significativa en los niveles macro y micro y en su interacción, pasando constantemente del análisis a la síntesis y de la síntesis al análisis.

Gran parte de la investigación de Ezequiel en estos proyectos es autobiográfica, a menudo en forma de historias orales. La integración de las historias, del poder y de la sabiduría de las personas produce procesos de transformación muy poderosos. La investigación que nos propone tiene un enfoque decididamente biográfico y cooperativo:

Mucha de nuestra investigación es autobiográfica, historias orales, porque es la más efectiva y necesaria de las fuentes primarias... Así que todo lo que escribimos es autobiográfico. Todo. Nos situamos en esta perspectiva porque no creemos que se pueda hacer nada con objetividad. La mayor parte de nuestro trabajo es en torno a las historias de la gente, el poder del lugar y la sabiduría de la gente. Cuando los juntamos, comienzan a ocurrir procesos ecológicos realmente interesantes, y lo vemos [...] Nos dimos cuenta, por todas las lecturas que habiamos hecho, que la investigación nunca fue una empresa aislada o singular. Todo nuestro enfoque ontológico de la investigación ha sido colaborativo.

Colocar a los sujetos en el centro del proceso implica, manifiesta Ezequiel, apoyar una epistemología constructivista e interpretativa, incompatible con el mito de la objetividad, y orientada por la plática o diálogo como eje vertebrador. En este contexto, es importante redefinir y ampliar el significado del conocimiento:

Así que el diálogo no es sólo un resultado, sino que es un proceso, y el proceso es un resultado en sí mismo. $Y$ dentro de ese resultado tienes la investigación, tienes la pedagogía, y tienes la construcción de la comunidad... Esto una oportunidad para un tipo diferente de explosión de energía, de ideas, de posibilidades, ¿verdad? Y lo que hacemos también es enfatizar diferentes perspectivas privilegiar del conocimiento: el conocimiento de la cognición, pero también el conocimiento de la emoción, el conocimiento de las relaciones y el conocimiento del cuidado.

Ezequiel defiende una perspectiva crítica que trasciende los aspectos cognitivos y se convierte en una actitud política y cultural que promueve la diversidad y el desorden. El diálogo crítico entre los diferentes grupos es muy importante en el trabajo educativo y político. El buen aprendizaje, tal como nos lo enseñan los antropólogos culturales, dice Ezequiel, es principalmente social; en segundo lugar, el buen aprendizaje es cognitivo; y finalmente, es una cuestión técnica. Ezequiel apoya la necesidad de promover la capacidad de los profesores para pensar y crear conocimiento.

Este líder comunitario se percibe a sí mismo como un académico en una posición delicada con respecto a su estatus. Reconoce la 
necesidad de innovación en la universidad, promoviendo procesos educativos grupales $\mathrm{y}$ el empoderamiento de los participantes. Promueve programas innovadores que promueven la autoconciencia, la agencia y el aprendizaje alternativo, en contraste con los enfoques convencionales. En particular, se destacan los esfuerzos para ampliar la participación en la universidad de los estudiantes vulnerables o pertenecientes a minorías desfavorecidas que trabajan con familias y grupos de la comunidad local. Por otro lado, Ezequiel critica el tradicionalismo de las universidades, que siguen reproduciendo modelos muy tradicionales de aprendizaje y gestión, y que no son capaces de responder adecuadamente a los cambios sociales y culturales de las últimas décadas.

En este campo, Ezequiel propone una redefinición completa de la formación inicial del profesorado, con el objetivo de adaptar los modelos de enseñanza y aprendizaje universitarios a las necesidades de los futuros educadores, así como de los intelectuales y críticos innovadores. Su visión es muy crítica de un conocimiento y una investigación que se considera objetiva, neutral y distante. Por otro lado, aboga por una epistemología basada en el constructivismo social. Considera que las universidades corren el riesgo de convertirse en instituciones sin importancia en las próximas décadas si no son capaces de reconfigurar los métodos de enseñanza y aprendizaje y las condiciones para la transmisión del conocimiento:

La parte radical de esto es que la universidad va a necesitar reformular lo que es la enseñanza, el aprendizaje, el liderazgo y la construcción del conocimiento. Creo que, personalmente, si continuamos en el camino que hemos recorrido, la universidad se volverá irrelevante y morirá, tal como la conocemos. Creo que, al igual que las escuelas públicas, ha habido un ataque neoliberal a las instituciones públicas, y ese ataque no se ha detenido con la enseñanza secundaria, ha llegado hasta las universidades.
Historias de vida en contextos migratorios y de movilidad académica: contribuciones y oportunidades

La historia de vida personal y profesional de Ezequiel nos lleva a considerar varias cuestiones centrales en relación con las cuestiones del aprendizaje y de la diversidad intercultural:

- La primera cuestión se refiere a la importancia de la cooperación, la comunidad y el grupo. Frente al modelo dominante, que enfatiza la competitividad y el logro de objetivos individuales, es importante fomentar un enfoque colectivo dentro de las instituciones educativas, pero también en contextos comunitarios y ciudadanos. Los valores comunitarios son necesarios para crear contextos apropiados para el desarrollo y la formación. Esto incluye la mejora de las relaciones entre las escuelas y los contextos locales, con miras a promover comunidades de aprendizaje. Además, la construcción de vínculos sociales y de grupo puede ser un factor importante para compensar la falta de capital social, especialmente en el caso de individuos y grupos desfavorecidos o vulnerables.

- Los contextos transfronterizos y multilingües constituyen ecosistemas de gran complejidad sociocultural. Estos contextos ofrecen muchas oportunidades para desarrollar experiencias y relaciones que pueden ayudar a mejorar la tolerancia, la gestión del choque cultural, la comprensión de las diferencias, la aceptación de la diversidad intercultural, la flexibilidad y la gestión de la identidad compartida. En la narrativa aquí presentada encontramos elementos políticos, culturales y metodológi- 
cos para profundizar la construcción de una eco-pedagogía del cruce fronterizo.

- Una pedagogía itinerante, nómada y vivencial debe basarse en el diálogo como principio central. Las contribuciones de Célestin Freinet y Paulo Freire, entre otros, pueden ayudarnos a identificar enfoques basados en el diálogo y el intercambio (FREINET, 1971; FREIRE, 1996). En esta perspectiva dialógica, el conocimiento se construye en la interacción, tiene un carácter contextual y sirve para transformar la realidad. Las historias de vida en formación y las metodologías autobiográficas nos muestran una forma de trabajar en la formación basada en la colaboración y con el objetivo de fomentar el desarrollo del conocimiento y del empoderamiento colectivos.

- Es necesario adoptar una perspectiva histórica y comparativa. Este enfoque debería permitirnos situar los procesos culturales y educativos, las experiencias de formación y los itinerarios biográficos dentro de marcos sociales más amplios. Las universidades acogen ahora a un nuevo público de "estudiantes no tradicionales" de origen popular, a veces sin una perspectiva histórica que permita contextualizar mejor los procesos de transformación. La migración y la movilidad académica son parte de estos procesos que debemos entender en su compleja y cambiante dinámica temporal.

- Las universidades, al igual que otras instituciones de educación formal, tienden a reproducir modelos de gestión y aprendizaje tradicionales y anticuados. La migración plantea importantes desafios a la educación superior. Frente a instituciones que históricamente han dado la espalda a la diversidad cultural, el estudio de caso de este artículo nos muestra la necesidad de desarrollar y profundizar un enfoque basado en la inclusión social para favorecer los itinerarios formativos de los estudiantes no tradicionales, vulnerables y desfavorecidos. Esta tarea es un reto importante que requiere enfoques innovadores en los ámbitos de los contenidos curriculares y de los métodos de aprendizaje.

- Contra los abusos autoritarios, xenófobos y exclusivos desarrollados en Europa, América y otras zonas geográficas, necesitamos iniciativas de resistencia colectiva y lucha organizada para construir alternativas basadas en la igualdad, el pluralismo, la tolerancia, la cooperación y el respeto de los derechos humanos.

- El creciente uso de enfoques autobiográficos e historias de vida en la investigación sobre viajes, migración y movilidad cumple diferentes funciones:

- Promueve la producción de conocimientos: desarrollo del conocimiento de los procesos migratorios y de la compleja dinámica del cambio sociocultural y de identidad.

- Sensibiliza sobre los problemas que plantea este fenómeno y promueve la comprensión cruzada: las historias de vida de los migrantes y sus comunidades (familias, grupos generacionales) promueven una mejor comprensión del punto de vista de los demás, facilitan la superación del etnocentrismo y aumentan el nivel de tolerancia (LECHNER, 2018).

- Permite contextualizar las situaciones y comparar diferentes contextos: sociedades de origen y de acogida, generaciones en diferentes momentos del 
ciclo vital, diferentes grupos étnicos y culturales que han emigrado dentro de un mismo país o región, comparación de un mismo grupo cultural desde un punto de vista diacrónico.

- Promueve un buen enfoque de intervención y formación: los profesionales implicados en el apoyo y el asesoramiento de los inmigrantes y las personas en movilidad necesitan saber más sobre este tema, desde una perspectiva científica y de matices. Esto puede facilitar un mejor posicionamiento profesional por parte de los profesionales de la salud, los servicios sociales, la policía, el poder judicial, los profesores, los formadores, los psicólogos, los consejeros laborales y los mediadores interculturales.

- Proporciona un buen nivel de teorización y problematización: el objetivo de la investigación es también producir teorías que nos ayuden a explicar y comprender mejor los procesos interculturales. Las historias de vida son un enfoque útil para explorar, a nivel meso y micro, los temas relacionados con los viajes, la movilidad y la migración, así como las relaciones entre estos niveles y los elementos macroestructurales, tales como la clase social, el género, la etnia y el grupo de edad.

\section{Las historias de vida de} inmigrantes académicos como enfoque para desarrollar la inclusión social y la diversidad intercultural

La investigación biográfica puede contribuir al estudio de las identidades fluidas, cambiantes y heterogéneas de la modernidad tardía (BAUMAN, 2000). Para ello, las biografias deben ser consideradas como formadas y condicionadas histórica y socialmente. Las transformaciones de la identidad biográfica dependen de los recursos biográficos del individuo entrevistado, concebidos como experiencias y conocimientos acumulados a lo largo del ciclo vital. De esta manera, el investigador podrá generar mejor sus estrategias de interpretación y hacer frente a las diferencias culturales y los conflictos que puedan surgir en la historia de la vida. Prestará más atención a la relación entre "narrador" y "narrador", a las diferentes oportunidades biográficas de la historia, a las condiciones en las que se encuentra el entrevistado en el momento de la historia. El análisis biográfico permite explorar los diferentes discursos del narrador, las estructuras de significado y los modelos de creencias que el sujeto establece; estos discursos están condicionados culturalmente y conducen a la construcción de una identidad narrativa personal, pero enraizados en un contexto social e histórico particular (GONZÁLEZ-MONTEAGUDO, 2016). El caso presentado en este artículo presenta las posibilidades de un enfoque experiencial, biográfico y cualitativo de las experiencias de migración y movilidad académica desde la perspectiva de un docente e investigador comprometido que ha teorizado y practicado la narrativa como dimensión fundamental de la formación, el desarrollo local y la construcción de una identidad tolerante y abierta, en el contexto de un mundo conectado y globalizado (BRETON, 2014; KIM, 2014).

En los nuevos contextos globalizados, posmodernos y cambiantes, las historias de vida y los relatos -como prácticas multiformes de investigación, formación, intervención social, testimonio histórico y construcción de identidades- aspiran a profundizar una forma de trabajo integradora y compleja, para favorecer el desarrollo de la autonomía personal, la convivencia tolerante y la participación social. 
Los enfoques narrativos biográficos, ya sean de investigación, de formación o de intervención social, pretenden conectar el pasado, el presente y el futuro en una perspectiva proyectiva.

Para estudiar la identidad (inter)cultural, la trayectoria migratoria, la adaptación a la sociedad de acogida, la formación y la integración social de las personas de origen inmigrante, creemos que debemos de defender una perspectiva de investigación antirracista y no eurocéntrica, guiada por los valores de la justicia y de la igualdad.

Actualmente, y debido al clima político y social que atraviesa Europa, estas posiciones parecen casi revolucionarias, pero en realidad derivan de los derechos humanos y de los valores reconocidos en las constituciones de los distintos países europeos. El enfoque narrativo propuesto en este texto permite un análisis profundo y empático de los problemas culturales y generacionales de los inmigrantes, a caballo entre la cultura de origen y la cultura de acogida. La lengua, la gestión del espacio y del tiempo, las relaciones familiares e interpersonales, los métodos educativos, el trabajo y la autoridad son dimensiones importantes de la cultura, a las que debemos prestar atención si queremos promover una integración social, ciudadana y laboral que respete las identidades y experiencias culturales de las personas inmigrantes (AMORÓS-TORRÓ, 2018).

La investigación biográfica y narrativa nos permite comprender la experiencia de la inmigración de una manera que no parece posible obtener con otros métodos. En primer lugar, el método biográfico nos permite recoger de primera mano la experiencia migratoria de los migrantes, que llevan a cabo un trabajo continuo de revisión de su identidad y de autoconocimiento sobre su propio proceso de aculturación e integración en la sociedad de acogida.
Los inmigrantes nos muestran cómo están viviendo el proceso de aculturación, que implica un incesante proceso de negociación consigo mismos y con el entorno, y cómo están llegando a diferentes momentos de síntesis y de hibridación, entre los universos culturales de origen y de acogida (AMORÓS-TORRÓ, 2018). Sus historias de vida nos involucran en estos procesos y nos permiten contribuir a la recreación y reconstrucción de la identidad por medio de las narrativas biográficas. Los costes de las negociaciones internas, el sentimiento de lealtad hacia la comunidad cultural de origen, la necesidad de integración y adaptación al nuevo marco de referencia, son sólo algunos de los procesos a los que se ven obligados los menores de origen inmigrante.

Otra de las contribuciones que ofrece la investigación biográfica y narrativa en el caso de los inmigrantes es la capacidad de captar la temporalidad y la experiencia del tiempo que tienen estas personas. La vivencia del tiempo personal se sitúa en el contexto del tiempo histórico y social. Como muchos autores han descrito (BERTAUX, 1997; FERRAROTTI, 1983), en el conocimiento biográfico el tiempo biográfico-personal (la temporalidad como vivencia subjetiva) está entrelazado con el tiempo histórico-social. Esto último concierne a grandes grupos de personas e implica cambios decisivos en la vida de las personas (movimientos migratorios, catástrofes, guerras y acontecimientos similares).

La perspectiva biográfica se centra en la persona, humaniza la investigación y le da sentido, anclándola en la experiencia. De esta manera, adquiere una legitimidad que resulta completamente diferente de la generalización o la racionalidad que requieren otros tipos de enfoques (BRUNER, 1991). Es una legitimidad que surge de la experiencia humana, derivada de vivir el fenómeno de la inmigración en un tiempo y un espacio concretos. $Y$ no hay otros 
modos de conocer todo esto que puedan igualar la narración de la experiencia en primera persona. Esta es una de las grandes riquezas y desafios de la investigación biográfica y narrativa (GONZÁLEZ-MONTEAGUDO, 2008; FERRAROTTI, 1983; LAINÉ, 1996). Esta contribución es particularmente significativa en los estudios sobre los movimientos migratorios y las relaciones interétnicas, ya que la investigación centrada en las personas hace visibles las experiencias humanas ocultas tras una visión de la inmigración en la que habitualmente sólo prevalecen los datos cuantitativos o las teorías políticas y sociológicas.

Otro elemento positivo del método biográfico en contextos de migración es el contexto intercultural que se genera en el intercambio que la propia investigación biográfico-narrativa promueve y acompaña. La metodología biográfica se basa en gran medida en la entrevista biográfica, que posee las características de una conversación entre dos personas (investigador y narrador), en donde la horizontalidad y el intercambio entre iguales son esenciales para el desarrollo de la metodología. El investigador (que tal vez puede ser, al mismo tiempo, un educador o un trabajador social) y el narrador tienen diferentes marcos culturales y universos simbólicos. Esto implica que las formas en que ambos darán sentido a la realidad pueden no sólo diferir, sino también ser contradictorias. El intercambio comunicativo entre estas dos personas transforma el espacio metodológico en un espacio intercultural y existencial, ya que la metodología requiere un posicionamiento hermenéutico que aspira a comprender el mundo del otro (LECHNER, 2018). La entrevista biográfica y la historia de la vida son, en sí mismas, un texto nacido de la experiencia intercultural, un espacio de mediación entre dos vidas que se comunican cara a cara.

Las narrativas biográficas de los inmigrantes nos confrontan con sus propios mar- cos culturales (etnocentrismo) y requieren un ejercicio de distanciamiento que favorece el autoconocimiento. La conversación biográfica nos lleva a este ejercicio de descentración para acceder a la comprensión del otro, y este ejercicio enriquece claramente a investigadores y lectores de narrativas biográficas interculturales (LECHNER, 2018). A través de estas narrativas se accede también al universo del significado del otro. Este movimiento hermenéutico intercultural es posible gracias al método biográfico, tanto en el proceso metodológico como en el producto que constituye el texto resultante (BRUNER, 1991).

El reconocimiento constituye un aspecto fundamental de la aplicación del método biográfico. La dimensión ética y política del método biográfico se ha puesto a menudo de relieve bajo la perspectiva de "dar voz" a grupos e individuos que han sido generalmente excluidos de la historia oficial. En este sentido, la historia oral y el método biográfico nos permiten escuchar las historias de las minorias y de los grupos excluidos y silenciados.

Los inmigrantes viven entre dos culturas, la cultura de origen y la cultura de acogida. Los proyectos e itinerarios migratorios pueden ser analizados en términos de la interacción de estas dos culturas, con todas las contradicciones, esperanzas, riesgos, miedos e incertidumbres que derivan de la concepción y realización de la migración. El cruce de culturas dificulta un proceso que no es fácil en sí mismo. La fidelidad a la cultura de origen, así como la adaptación e integración en la cultura de acogida, requieren que los inmigrantes estén casi siempre involucrados en un proceso de negociación complicado y conflictivo.

En este sentido, la investigación narrativa, si bien tiene un interés real en la comprensión y la interpretación, debe prestar especial atención al conflicto y al análisis de una serie de dualismos que parecen constituir los esce- 
narios sociales y culturales actuales, entre los que se incluyen: la vida personal y pública, la identidad personal y sociocultural, la sociedad de origen y de acogida, la infancia y la edad adulta, el pasado y el futuro, los objetivos personales y los objetivos relacionados con el grupo familiar y cultural de origen.

\section{Bibliografía}

AMORÓS-TORRÓ, Marta. La dimensión espacial en la investigación biográfica. El « aquí » y el « allá » en los relatos de vida de migracións. Revista Brasileira de Pesquisa (Auto)Biográfica, Salvador, v. 03, n. 07, p. 21-34, 2018. Disponible en: https://www.revistas. uneb.br/index.php/rbpab/article/view/4481 Acceso en: 22 oct. 2019.

ARNOT, Madeleine, SCHNEIDER, Claudia, WELPLY, Oakleigh. Education, mobilities and migration: people, ideas and resources. Compare: A Journal of Comparative and International Education. London, 43 (5), p. 567-579, 2013. Disponible en: https://www.tandfonline.com/doi/ abs $/ 10.1080 / 03057925.2013 .822194$ ? journalCode $=c-$ com20 Acceso en: 22 oct. 2019.

BAUMAN, Zyzmunt. Liquid modernity. Cambridge: Polity Press, 2000.

BERTAUX, Daniel. Les récits de vie. Paris: Nathan, 1997.

BRETON, Hervé. L'expérience du voyage : une initiation à l'autoformation. Colloque sur l'Autoformation, Université de Strasbourg, 30 et 31 de octubre de 2014.

BRUNER, Jerome. Actos de significado. Madrid : Alianza, 1991.

CASTLES, Stephen, MILLER, Mark J. The Age of migration. International population movements in the modern world. New York: Palgrave Macmillan, $4^{\text {th }} \mathrm{ed}$, 2009.

CHANG, Aurora. Undocumented to Hyperdocumented: a jornada of protection, papers, and PhD status. Harvard Educational Review. Cambridge (Massachusetts), 81(3), p. 508-520, 2011. Disponib- le en: https://hepgjournals.org/doi/abs/10.17763/ haer.81.3.d84532vu27772424 Acceso en: 22 oct. 2019.

CHANG, Aurora. Priviledged and undocumented: Toward a Borderland Love Ethic. Association of Mexican-American Educators (AMAE) Open Issue. México DF, 9(2), p. 6-17, 2015. Disponible en: https:// www.redalyc.org/jatsRepo/3845/384555587004/ html/index.html Acceso en: 22 oct. 2019.

CLIFFORD, Valerie, MONTGOMERY, Catherine. Challenging conceptions of western higher education and promoting graduates as global citizens. Higher Education Quarterly. San Francisco, 68(1), p. 28-45, 2014. Disponible en: https://onlinelibrary. wiley.com/doi/abs/10.1111/hequ.12029 Acceso en: 15 oct. 2019.

DELGADO WISE, Raúl. Mexico-United States migration, 1980s-2010> In:n I. Ness (Ed.). The Encyclopedia of Global Human Migration. New York: Blackwell Publishing, 2013. p. 100-132.

DELGADO WISE, Raúl. Reflexiones sobre la cuestión migratoria México-Estados Unidos ante el triunfo electoral de Donald Trump. Migración y Desarrollo. México DF, 27, p. 156-167, 2016. Disponible en: https://issuu.com/comunicacionsocialuaz/docs/ mydv14n27 Acceso en: 15 oct. 2019.

DELGADO WISE, Raul, CHÁVEZ, Mónica. Migración calificada: entre la pérdida de talento y la oportunidad de transformar a México con innovación. Revista Mexicana de Política Exterior. México DF, 107, p. 117-135, 2016. Disponible en: http://www. scielo.org.mx/scielo.php?script=sci_abstract\&pi$d=S 1870-75992016000200167 \&$ lng $=$ es \& $n r m=i s o \quad$ Ac ceso en: 15 oct. 2019.

DELGADO WISE, Raúl, CHÁVEZ, Mónica, RODRíGUEZ, Héctor. La innovación y la migración calificada en la encrucijada: reflexiones a partir de la experiencia mexicana. Revista Interdisciplinar de Movilidades Humanas. Brasília, XXIV, 47, p. 153-174, 2016. Disponible en: http://www.scielo.br/scielo.php?pi$d=S 1980-85852016000200153 \&$ \&cript $=$ sci_abstrac t\&tlng=es Acceso en: 15 oct. 2019.

FERRAROTTI, Franco. Histoire et histoire de vie. La méthode biographique dans les Sciences Sociales. Paris: Librairie des Méridiens, 1983. 
FREINET, Célestin. La educación por el trabajo. México: FCE, 1971.

FREIRE, Paulo. La pedagogía de la esperanza. Madrid: Siglo XXI, 1996.

GONZÁLEZ-MONTEAGUDO, José. Des approches européennes non francophones en histoires de vie" (Note de synthèse). Pratiques / Analyses de Formation. Revue internationale. Paris, 55, p. 9-83, 2008.

GONZÁLEZ-MONTEAGUDO, José. Transitions et transformations des étudiants internationaux en contextes de mobilité académique. Pensée Plurielle. Bruxelles (Éditeur De Boeck Supérieur), 41(1), p. 111122, 2016. Disponible en: https://www.cairn.info/ revue-pensee-plurielle-2016-1-page-111.htm Acceso en: 15 oct. 2019.

KIM, Terry. Transnational academic mobility, internationalisation and interculturality. Intercultural Education. London, 20, p. 395-405. 2009a. Disponible en: https://www.tandfonline.com/doi/ abs/10.1080/14675980903371241 Acceso en: 15 oct. 2019.

KIM, Terry. Shifting patterns of transnational academic mobility: a comparative and historical approach. Comparative Education. London, 45(3), p. 387403. 2009b. Disponible en: https://www.tandfonline.com/doi/abs/10.1080/03050060903184957 Acceso en: 15 oct. 2019.

KIM, Terry. Transnational academic mobility, knowledge and identity capital. Discourse: Studies in the cultural politics of education. London, 31(5), p. 577591, 2010. Disponible en: https://www.tandfonline. com/doi/abs/10.1080/01596306.2010.516939 Acceso en: 15 oct. 2019.

KIM, Terry. The intellect, mobility and epistemic positioning in doing comparisons and comparative education. Comparative Education. London, 50(1), 58-72, 2014. Disponible en: https://www.tandfonline.com/doi/abs/10.1080/03050068.2013.874237 Acceso en: 15 oct. 2019.

KNIGHT, Jane. The changing landscape of higher education internationalisation - for better or worse? Perspectives: Policies and practice in higher education. San Francisco, 17(3), p. 84-90, 2013. Disponible en: https://www.tandfonline.com/doi/ab s/10.1080/01596306.2010.516939 Acceso en: 15 oct. 2019.

LAINÉ, Alex. Faire de sa vie una histoire. Paris: Desclée de Brouwer, 1996.

LECHNER, Elsa. Pesquisa biográfica no estudo de migrações: diálogos teórico-práticos no horizonte de uma utopia concreta. Revista Brasileira de Pesquisa (Auto)Biográfica, Salvador, v. 03, n. 07, p. 3544, 2018. Disponible en: https://www.revistas.uneb. br/index.php/rbpab/article/view/4950 Acceso en: 15 oct. 2019.

OLWIG, Karen Fog, VALENTIN, Karen. Mobility, education and life trajectories: new and old migratory pathways. Identities: Global Studies in Culture and Power. London, 22(3), 247-257, 2015. Disponible en: https:// www.tandfonline.com/doi/abs/10.1080/107 0289X.2014.939191 Acceso en: 15 oct. 2019.

SHELLER, Mimi. The new mobilities paradigm for a live sociology. Current Sociology Review. New York, 62 (6), p. 789-811, 2014. Disponible en: https:/ / journals.sagepub.com/doi/10.1177/0011392114533211 Acceso en: 15 oct. 2019.

SHELLER, Mimi, \& URRY, John. The new mobilities paradigm. Environment and Planning. London, 38(2), 207-226, 2006. Disponible en: https://journals.sagepub.com/doi/abs/10.1068/a37268 Acceso en: 15 oct. 2019.

UCKER, Lilian. A experiência de ser estudante internacional: discursos, práticas narrativas e aprendizagens em diálogo. Revista Brasileira de Pesquisa (Auto)Biográfica, Salvador, v. 03, n. 07, p. 257-272, 2018. Disponible en: https://www.revistas.uneb.br/ index.php/rbpab/article/view/4165 Acceso en: 15 oct. 2019.

URRY, John. Mobilities. London: Polity Press, 2007.

Recebido em: 15.11.2019 Revisado em: 01.04.2020 Aprovado em: 03.04.2020 
José González-Monteagudo Doutor em Pedagogia e professor titular da Faculdade de Educação da Universidade de Sevilha (Espanha). Foi professor convidado em universidades de Europa, América e Ásia. Pertence às associações francófona (ASIHVIF), anglófona (ESREA) e brasileira (BIOgraph) de pesquisa e formação biográficas. E-mail: monteagu@us.es

Wilton Carlos Lima da Silva Doutor em História (Universidade Estadual Paulista Júlio de Mesquita Filho, UNESP, 2000). Estágios de pesquisa nas Universidade de Coimbra (2010) e Sevilha (2015-2016). Professor Associado da Universidade Estadual Paulista Júlio de Mesquita Filho, UNESP. E-mail: wilton@assis.unesp.br 